Tropical Journal of Pharmaceutical Research October 2012; 11 (5): 815-821

(C) Pharmacotherapy Group,

Faculty of Pharmacy, University of Benin

Benin City, 300001 Nigeria.

All rights reserved.

Available online at http://www.tjpr.org

Research Article

http://dx.doi.org/10.4314/tjpr.v11i5.16

\title{
Serological Prediction of infections in Diabetic Patients with Diabetes Ketoacidosis in Penang, Malaysia
}

\author{
Syed Wasif Gillani ${ }^{*}$, Syed Azhar Syed Sulaiman', Shameni Sundram², \\ Yelly Oktavia Sari ${ }^{3,4}$, Mirza Baig ${ }^{5}$ and Mian Muhammad Shahid Iqbal ${ }^{6}$ \\ ${ }^{1}$ School of Pharmaceutical Sciences, Universiti Sains Malaysia, Pulau Pinang, Malaysia, ${ }^{2}$ Doctor, Hospital Pulau \\ Pinang, 10990, Residential Street, Penang, ${ }^{3}$ Faculty of Pharmacy, Andalas University, Padang 25163, Indonesia, \\ ${ }^{4}$ Discipline of Clinical Pharmacy, School of Pharmaceutical Sciences, Universiti Sains Malaysia, 11800 Penang, \\ ${ }^{5}$ Department of Clinical Pharmacy, Aimst University, Kedah, ${ }^{6}$ School of Pharmacy and Health Sciences, International \\ Medical University, Malaysia.
}

\begin{abstract}
Purpose: To determine the prevalence and predictors of infection in diabetic patients with diabetic ketoacidosis (DKA) who were $\geq 18$ years.

Methods: A retrospective cohort design was adopted for this study. A total of 967 diabetes ketoacidosis patients from Hospital Pulau Pinang for the 3-year period, Jan 2008 - Dec 2010, were identified and enrolled. The data were analysed, as appropriate, by Student t-test and ANOVA for the normally distributed data, Mann-Whitney U rank sum and Kruskall-Wallis tests for continuous, non-nominal data and Chi-square for dichotomous variables. Odd Ratios with 95\% confidence interval (Cl) were also presented where applicable.

Results: Of the total diabetes ketoacidosis patients, 112 (11.6\%) were cases without infection, 679 (70.2 \%) bacterial infection cases and 176 (18.2\%) presumed viral infection cases. The mean white blood count (WBC) for all the patients was 18,177 \pm 9,431 while 721 (74.6\%) had leukocytosis, defined by $W B C \geq 15,000 / \mathrm{mm}^{3}$. WBC differential, leukocytosis, as well as sex and body temperature were not significant predictors $(p>.05)$ of bacterial infection. There was, however, a significant difference $(p<.05)$ in terms of age within groups, as those $>57$ years showed a higher rate of infection.

Conclusion: The infection rate in elderly patients with DKA is high and a majority of them lack clinical evidence. Age has a significant effect on the rate and prediction of infection. Leukocytosis is commonly found but severe ketoacidosis was more likely than the presence of infection.
\end{abstract}

Keywords: Diabetes mellitus, Diabetes ketoacidosis, Infections, Predictors, White blood cells 


\section{INTRODUCTION}

It is commonly believed that acute infectious illness can precipitate episodes of ketoacidosis in patients with diabetes mellitus. For this reason, a diligent search for bacterial infection is considered essential in the initial evaluation of patients with diabetes ketoacidosis (DKA). Together with hyperglycemic coma, diabetes ketoacidosis (DKA) is the most severe acute metabolic complication of diabetes mellitus [1]. Defined by the triad, hyperglycemia, acidosis, and ketonuria, DKA can be inaugural or complicate known diabetes [2]. Although DKA is evidence of poor metabolic control and usually indicates an absolute or relative imbalance between the patient's requirements and the treatment, DKA-related mortality is low among patients who received standardized treatment, which includes administration of insulin, correction of hydroelectrolytic disorders, and management of the triggering factor (which is often cessation of insulin therapy, an infection, or a myocardial infarction) [3-6].

Unfortunately, the sign and symptoms associated with the ketoacidotic state often make the search for potential infections difficult. These patients often appear ill, and may have non-specific symptoms including malaise, headache, abdominal pain, vomiting and altered mental status [9]. Consequently, investigators have searched for predictors of infection, such as the presence of leukocytosis or abnormal differential, in patients with DKA. Slovis et al [7] reported that an elevated band neutrophil count could reliably predict the existence of serious occult bacterial infections in adults with DKA. However, the majority of adult studies have failed to show a clear association between the presence of leukocytosis and the existence of infections in the patient population [6].

Although there is no proof that diabetics are more susceptible to infection, they seem to have more difficulty handling infection once it occurs [8]. Indeed, several aspects of immunity are altered in diabetic patients: polymorphonuclear leukocyte function is depressed, particularly when acidosis is present, and leukocyte adherence, chemotaxis, phagocytosis and bactericidal activity may also be impaired [9]. Joshi et al [10] reported the lack of clinical evidence that diabetic patients are more susceptible to infection that nondiabetic patients. Nevertheless, infection is a well-recongnized trigger of DKA, but studies in non-diabetic patients have consistently shown a relationship between the presence of leukocytosis and the existence of bacterial infections [11,12].

Since no large scale study regarding DKA and infection in the diabetic population have been reported, the objective of this study was to determine the prevalence of infection in diabetic adults with ketoacidosis and to evaluate presenting clinical features that predict the infection. Specifically, the absolute leukocyte count, cell differential, and the presence of leukocytosis were selected as the primary variables of interest. In addition, age, sex, body temperature and new onset were evaluated as potential predictors of infection.

\section{METHODS}

The study was conducted in an urban government-owned hospital in Penang Malaysia. The records of patients $\geq 18$ years, who were diagnosed and admitted for diabetes mellitus with DKA between Jan 1, 2008 and December 31, 2010 were reviewed. The charts were identified by the International Classification of Diseases (ICD-9) admission and/or discharge codes of "diabetes ketoacidosis' and 'diabetes mellitus'. In this review, DKA was defined by an elevated serum glucose $\geq 250 \mathrm{mg} / \mathrm{dL}$, a serum bicarbonate $\leq 15 \mathrm{meg} / \mathrm{L}$ and $\mathrm{pH} \leq 7.35$.

The entire medical record was reviewed for each hospital admission. Clinical evaluations, including a search for potential infections, as 
well as all treatment decision, were conducted at the discretion of the admitting physicians. Demographic characteristics were recorded for each patient in addition to the initial maximum body temperature, serum glucose, serum bicarbonate, venous or arterial $\mathrm{pH}$, total leukocyte count and cell differential. Fever was defined as a temperature $\geq 38{ }^{\circ} \mathrm{C}$; the method by which temperature was measured was not recorded. Clinical definition of leukocytosis is when there is a total blood cell count of $\geq$ $15,000 / \mathrm{mm}^{3}$. All admissions were included in the analysis, including those with missing data.

The following a priori definitions regarding infection were applied: patients with no history (e.g., respiratory symptoms, diarrhea), physical examination (e.g., otitis media, cellulitis), rapid streptococcal testing, culture and/or radiographs, during the entire hospital course, were defined as having no infection. Patients with the documentations of symptoms, signs and/or radiographs consistent with upper and/or lower respiratory tract infections, for which no antibiotics were given, were defined as having presumed viral infection [9]. Patients treated with antibiotics, as deemed necessary by the treating physician, were defined as having bacterial infection. So patients were categorized into 3 groups based on these definitions; (1) no infection; (2) presumed viral infection; and (3) bacterial infection. Comparison was made between these groups and are stated as "intergroup comparisons".

This study was approved by the hospital's "Clinical Research Committee (CRC)" as well as the Ministry of Health Malaysia (MOH).

\section{Statistical analysis}

For primary purpose of descriptive analyses, the patients with bacterial infection were further divided into minor and major infection categories based on the likelihood of serious sequel as evidenced in the diabetes literature $[1,5,9,12-14]$. Statistical analyses were performed using SPSS version $17 \AA$. Analyses included the use of student t-test and ANOVA for the normally distributed data, the Mann-Whitney $U$ rank sum and KruskallWallis tests for continuous, nonnominal data and chi-square for dichotomous variables. When evaluating an association between 2 continuous variables, such as total white blood cell count and pH, Pearson's correlation test was applied. All reported $p$ values are 2-tailed and significance was established at $p \leq .05$. Regression analysis was applied where there was significant association among variables and outcome. Data are presented as mean \pm standard deviation (SD) unless otherwise indicated. Odds ratios with $95 \%$ confidence intervals $(\mathrm{Cl})$ are presented where applicable. Power calculations using measured proportions were made using Sample Power, version 1.00 (SPSS, Chicago, IL).

\section{RESULTS}

During the period of January 2008 through December 2010, there were a total 2174 diabetes patients admitted; all 2174 (100\%) patients' charts were reviewed. Of these 967 $(44.5 \%)$ admissions were for DKA. The mean age of the patients was $37.9 \pm 5.7$ years; $48.9 \%$ were male. The mean admission glucose was $551 \pm 170 \mathrm{mg} / \mathrm{dL}$ whereas the mean bicarbonate was $8.5 \pm 3.7$ $\mathrm{meg} / \mathrm{L}$ and mean $\mathrm{pH} 7.15 \pm 0.14$. The mean admission white blood cell count was 18,177 $( \pm 9,431) ; 721(74.6 \%)$ had leukocytosis. The mean maximum body temperature was 37.2 $\pm 4.0^{\circ} \mathrm{C} ; 57.1 \%$ had fever.

Table 1 summarizes the demographic characteristics of the 3 groups. Among the 967 admissions, 112 (11.6 \%) had no evidence of infection. There were 176 with presumed viral infection (18.2\%), and 679 with bacterial infection $(70.2 \%)$. Of those with bacterial infections, 453 had minor infections (66.7\%), and 226 had major infections (33.3 \%). 
Table1: Presenting clinical symptoms for infection category among patients with DKA (mean \pm SD)

\begin{tabular}{lllll}
\hline Parameter & No infection & Viral infection* $^{*}$ & Bacterial infection $^{\text {Total }}$ \\
\hline Patients & $112(11.6 \%)$ & $176(18.2 \%)$ & $679(70.2 \%)$ & $967(100 \%)$ \\
Age (years) & $32.1( \pm 3.2)$ & $35.7( \pm 4.6)$ & $42.1( \pm 7.5)$ & $37.9( \pm 5.7)$ \\
Temperature (C) & $37.2( \pm 4.7)$ & $37.4( \pm 0.97)$ & $37.1( \pm 0.84)$ & $37.2( \pm 4.0)$ \\
Glucose (mg/dL) & $571( \pm 147)$ & $640( \pm 168)$ & $627( \pm 197)$ & $551( \pm 170)$ \\
$\mathrm{HCO}$ (meq/L) & $8.7( \pm 3.5)$ & $8.4( \pm 3.3)$ & $8.5( \pm 3.8)$ & $8.5( \pm 3.7)$ \\
$\mathrm{pH}$ & $7.15( \pm 0.13)$ & $7.16( \pm 0.15)$ & $7.16( \pm 0.11)$ & $7.15( \pm 0.14)$ \\
$\mathrm{WBC} / \mathrm{mm}^{3}$ & $17,964( \pm 10,104)$ & $17,773( \pm 8,420)$ & $17,291( \pm 9,724)$ & $18,177( \pm 9,431)$ \\
$\mathrm{ANC} / \mathrm{mm}^{3}$ & $13,781( \pm 9,457)$ & $12,645( \pm 8,974)$ & $11,964( \pm 7,990)$ & $13,249( \pm 8,000)$ \\
$\mathrm{ABC} / \mathrm{mm}^{3}$ & $747( \pm 1,345)$ & $661( \pm 1,397)$ & $793( \pm 2,343)$ & $741( \pm 1,574)$ \\
$\mathrm{ALC} / \mathrm{mm}^{3}$ & $3,038( \pm 2,921)$ & $3,455( \pm 2,752)$ & $2,990( \pm 1,737)$ & $3,227( \pm 2,771)$ \\
New DM & $60(53.6 \%)$ & $73(41.5 \%)$ & $221(32.5 \%)$ & $416(43.0 \%)$ \\
Male:Female $(\%)$ & $48.2 \%: 51.8 \%$ & $47.3 \%: 52.7 \%$ & $51.2 \%: 48.8 \%$ & $48.9 \%: 51.1 \%$ \\
\hline
\end{tabular}

Abbreviations: WBC = white blood cell count, ANC, absolute neutrophil count; ABC, Asbolute band count; ALC = absolute lymphocyte count; * Presumed. DM = diabetes mellitus

Table 2: Severe bacterial infections in adult DKA patiebts (mean \pm SD)

\begin{tabular}{llll}
\hline Patients $^{*}, \mathbf{N}(\%)$ & Age (years) & WBC $\mathbf{m m}^{3}$ & Infection* $^{*}$ \\
\hline $245(36.1)$ & $47.6 \pm 3.1$ & $31,200 \pm 5,678$ & Septicemia \\
$212(31.2)$ & $41.7 \pm 4.5$ & $25,712 \pm 5,310$ & Community acquired pneumonia \\
$117(17.2)$ & $39.2 \pm 2.9$ & $15,180 \pm 6,873$ & Hospital acquired pneumonia \\
$198(29.2)$ & $41.5 \pm 4.7$ & $14,890 \pm 4,790$ & Ecoli urinary tract infection \\
$359(52.9)$ & $49.1 \pm 5.6$ & $18,310 \pm 7,851$ & Cellulitis of leg (toe) \\
${ }^{*}$ A majority of patients had multiple infections; hence, the total number of patients exceeded 100 \% (679)
\end{tabular}

Minor bacterial infections included otitis media, streptococcal pharyngitis, sinusitis, and bronchitis. Major bacterial infection included pneumonia, septicemia, cellulitis (leg) and urinary tract infection. The demographic characteristics of those with major bacterial infections are shown in Table 2.

There was no significant difference in maximum temperature in the within group comparison. The presence of fever, however, was a significant predictor of infection as 70 $\%$ of those with fever had infection whereas $25 \%$ who were without fever had infection ( $p$ $<.001)$. On the other hand, fever was not a significant predictor of bacterial infection. In reviewing the data, a significant correlation was found between the total white blood cell count and the $\mathrm{pH}(\mathrm{r}=-.57, p<.001)$. There was also a significant correlation when WBC was compared with serum bicarbonate $(r=$ $.44, p<0.001)$. Furthermore, when dichotomizing $\mathrm{pH}$ around the mean $\mathrm{pH}$ (i.e., $\mathrm{pH} \leq 7.15)$, a significant association was found between leukocytosis and $\mathrm{pH}$; the odd ratio of this comparison was $2.67(95 \% \mathrm{Cl}=$ 1.85 to $3.19, p<.001)$. Finally, when comparing those with serum bicarbonate concentration above and below the mean value of $8.6 \mathrm{meq} / \mathrm{L}$, a significant relationship was found between leukocytosis and bicarbonate $($ odds ratio $=1.97,95 \% \mathrm{Cl}=1.47$ to $2.51, p<.001)$.

Logistic regression is not applicable as there were no significant intergroup differences in age, sex, temperature, new onset, total white blood cell count, total neutrophil count, total band count and total lymphocyte count. While comparison in-between, both with leukocytosis $(\mathrm{n}=721)$ versus those without $(\mathrm{n}=246)$; similarly, there was also no significant intergroup differences. Furthermore, when attempting to use leukocytosis as a predictor of bacterial infection, no significant differences could be found. The power of the analytical comparison of bacterial infection and leukocytosis was $47 \%$ (based on the sample size of 721 and 246 for the 2 groups and the measured difference in proportions of .07). Notably, of the 80 
patients with initial white blood cell counts greater than $40,000 / \mathrm{mm} 3$, only 27 (33.8\%) had a documented evidence of infection during the hospital course (Table 2). This study suggests that all the predicting factors such as temperature, WBC, neutrophil count, total blood count and total lymphocyte count, are not predicting markers for infections among the patients with diabetes ketoacidosis.

The power of analysis of major bacterial infection and leukocytosis was $17 \%$ (based on the measured difference in proportions of 0.02). Finally, when comparing those with leukocytosis to those with any infection, viral or bacterial, no significant association could be found. The power of this analysis was 15 $\%$ (based on measured difference in proportions of .05).

Using the same analytical approach as above, no significant relationship could be found between any category of cell differential and bacterial infection (Table 1). Furthermore, when comparing the categories of the cell differential to the presence or absence of any infection, no significant relationship could be found. There was, however, a significant $(p<.05)$ difference in age between the 3 groups, as those aged $>$ 57 years had a higher rate of infection than $\leq$ 57 years. When age was compared with those with and without bacterial infections, only a trend towards significance was found $(p=.1)$. Further analysis showed that $70.8 \%$ $(481 / 679)$ of the patients $\leq 57$ years of age had bacterial infections compared with 29.1 $\%(198 / 679)$ of the patients > 57 years; the odds ratio for this comparison was 2.5 (95 $\% \mathrm{Cl}=1.48$ to $4.31, p=.03$ ).

\section{DISCUSSION}

Early detection of bacterial infection in patients with diabetes mellitus is a clinical priority. This need is based on the higher morbidity associated with infections in adults with diabetes. Impaired host responses may be responsible for this increased severity of infection. For example, it has been shown that polymorphonuclear leukocytes in diabetic patients, particularly when acidosis is present, may have defects in adherence, chemotaxis, phagocytosis and antioxidant activity involved in bactericidal function [9]. Moreover, diabetic patients with ketoacidosis have alterations in monocyte receptor functions [13]. Finally, a decreased responsiveness of T-cell lymphocytes to mitogenic stimulation has been shown [14].

Despite these concerns, studies have failed to identify reliability predictors of infection in the most severe diabetics, namely those with ketoacidosis. Guerin et al [15] evaluated 140 adults with DKA and concluded that total WBC, blood glucose and bicarbonate had little or no value in predicting infection. Slovis et al [7] attempted to identify adults with occult bacterial infections by examining the records of 169 patients with DKA. Findings showed that $30(21 \%)$ of the patients admitted had occult infections (11 minor infections and 19 major infections) that were not diagnosed initially but within the first 48 hours of administration. Solvis concluded that, of all the variables examined, only a band neutrophil counter greater than 10\% could reliably predict major occult bacterial infections in adult patients with DKA. Despite the findings of Solvis et al [7], findins from the present study suggest that both neutrophil count and WBC count are not predictors of infection among diabetes ketoacidosis patients. On comparison of blood differentials against confirmed infection versus no infection, it is evident that blood cell differentials are falsely elevated and not a true predictor/marker of infections among DKA patients.

On the other hand, the association between DKA and leukocytosis in the absence of infection, predominantly in adult patients, is described in the literature. Golden et al [16] described 124 patients, aged 14-75 years, with moderate to severe DKA, nothing that the total WBC ranged from 4,200 to 48,000 . Dunger et al [17] found that the WBC count 
ranged from 5,100 to 43,000 in patients with severe DKA. The present study shows a range of 8,100 to 51,000 . The origin of the leukocytosis observed in adult patients with DKA is unknown but has been attributed, in part, to increased catecholamine release from the adrenal gland in response to stress; marked dehydration and resulting hemoconcentration may also play a role. On the other hand, kayashima et al [18] found that granulocyte colony stimulating factor does not appear to be a mediator in this response. It has been suggested that the leukocytosis is due to severeity of the DKA instead of infection marker in our study population.

This retrospective study attempted to identify the frequency of infection and then ascertain whether predictors of infection could be easily identified based on presenting data. Specifically, the patients were categorized into 3 groups based on infection type with high sample size so that comparison could be made with previously published studies on adult diabetes patients. We found that the majority of patients $69.5 \%$ had no clinical evidence of infection. Although $70.2 \%$ $(679 / 967)$ of the patients were treated with antibiotics, major bacterial infections were common, representing every third patient with severity of infection load, i.e., 226 (33.3 \%) of the adults with DKA. As shown in the power calculation, this high rate of bacterial infection, particularly of the major type, may have created a type I error in our analysis.

The findings on leukocyte counts in the range of 4,720 to 53,100 is inconsistent with other previous studies, possibly due to a higher sample size and also high mean age of the patients. There was no relationship, however, between leukocytosis or cell differential and infection in adults with DKA. Furthermore, 47 cases (17 major bacterial infections, 30 minor bacterial infections) of bacterial infections were not initially recorded by the admitting physician but noted within the first $48 \mathrm{~h}$; assuming that these were truly 'occult', they represented only $4.9 \%$ of the admission of the study which descriptively lower with the study done by Slovis et al [7] reported $21 \%$ occult bacterial infections. Furthermore, a band neutrophil count greater than $10 \%$, or any other portion of the cell differential, were not significant predictors of bacterial infection, including these occult infections, in our population. Again, the low prevalence of these infections is meaningful, but resulted in a underpowered analyses of small and unicentre study population.

Of clinical importance, we observed that patients > 57 years of age with DKA were more likely to have bacterial infections that those $\leq 57$ years. Yet, major infections were not more common in this group. In addition, statistically significant relationship between the $\mathrm{pH}$, the serum bicarbonate and the WBC were revealed in our post hoc analysis. This suggests that the observed leukocytosis may be another indicator of the severity of the ketoacidosis state rather than a predictor of infection.

\section{Limitations of the study}

There are other potential limitations of the study based on the design. A possible bias may be found in any retrospective chart review, particularly with identified dependent variables such as infection. As previously stated, the definitions of infection were applied a priori so as to capture every patient with a possible viral or bacterial infection. For instance, the presence of upper respiratory tract symptoms was taken as evidence of a presumed viral infection. This, in turn, may have resulted in an overestimation of the total number of viral infections in our analysis. On the other hand, because cultures, rapid streptococcal testing and chest radiographs were not performed on every patient, it is possible that the prevalence of bacterial infections was under-estimated. Furthermore, it likely that the prevalence of bacterial infections was over-estimated because all patients treated with antibiotics in the bacterial infection category were included. Also, it seems unlikely that a significant 
number of major bacterial infections were not appropriately identified (i.e., they were missed) when all 967 patients in this study were admitted and the cases reviewed.

Perhaps these same limitations in retrospectively identifying infections account for the wide range of infections rates in adults with DKA in previously published studies. For instance, Dunger et al [17] stated that infection, either major or minor, is listed as the cause of DKA in 15 to $50 \%$ of the cases. Our findings of $47.7 \%(n=461)$ infection rate is within the wide range reported.

\section{CONCLUSION}

The infection rate in elderly patients with DKA is high and majority of them did not show clinical evidence. Age has a significant effect on the rate and prediction of infection. Leukocytosis is commonly present but more likely, it reflects the severity of ketoacidosis rather than the presence of infection.

\section{REFERENCES}

1. Umpierrez GE, Khajavi $M$, Kitabchi AE. Diabetic ketoacidosis and hyperglycemic hyperosmolar syndrome. Am J Med Sci 1996; 311: 255-263.

2. Kitabchi AE, Nyenwi EA. Hyperglycemic crisis in diabetes mellitus: diabetes ketoacidosis and hyperglycemic hyperosmolar state. Endocrinol Metab Clin N Am 2006; 35: 725-751.

3. Wagner A, Risse A, Brill HL, Wienhausen-Wilke V, Rottmann $M$, Sondern $K$, et al. Therapy of severe diabetic ketoacidosis. Zero-mortality under very-low-dose insulin application. Diabetes Care. 1999; 22: 674-677.

4. Kitabchi AE, Wall BM. Diabetic ketoacidosis. Med Clin North Am 1995; 79: 9-37.

5. Davoren $P$. Precipitating factors in diabetic ketoacidosis. Med J Aust 1991; 154: 855-856.

6. Foster DW, MacGarry JD. The metabolic derangement and treatment of diabetic ketoacidosis. N Engl J Med 1983; 309: 159169.

7. Slovis CM, Mork VG, Slovis RJ, Bain RP. Diabetes ketoacidosis and infection: leukocyte count and differential as early predictors of serious infection. Am J Emerg Med 1987; 5: 1-5.

8. Hoffman WH, Burek CL, Walker JL, Fisher LE, Khichi $M$, Mellick LB. Cytokine response to diabetic ketoacidosis and its treatment, Clin. Immunol 2003; 108: 175-181.

9. Joshi N, Caputo GM, Weitekamp MR, Karchmer MW. Infections in patients with diabetes mellitus. $N$ Engl J Med 1999;3 41: 1906-1912.

10. Charfen MA, Fernandez FM. Diabetes Ketoacidosis. Emerg Med Clin North Am 2005; 23: 609-628.

11. Zukin $D D$, Garisham JE, Saulys A. Fever in Children. In: Rosen P, Barkin R, Danzi D, Eds. Emergency medicine: concepts and clinical practice, edn 4. Mosby, St. Louis, MO, Yearbook, 1998: 1088-1089.

12. Hamilton G, Anemia, Polycythermia. White blood cell disorders. In: Rosen P, Barkin R, Danzi D, Eds. Emergency Medicine; concepts and clinical practice. eds. Mosby, St. Louis, MO, Yearbook, 1998:2072-2073.

13. Steward J, Collier A, Patrick AW, Clarke BF, Weir $D M$. Alterations in monocyte receptor function in type 1 diabetic patients with ketoacidosis. Diabetic Medicine. 1991; 8: 213-16.

14. Lebovitz HE. Diabetes Ketoacidosis. Lancet 1995: 345: 767-772.

15. Guerin JM, Meyer P, Segresta JM. Hypothermia in diabetes ketoacidosis. Diabetes Care 1987; 10: $801-802$.

16. Golden SH, Peart VC, Kao WH, Brancati FL. Perioperative glycaemic control and the risk of infections complication in a cohort of adults with diabetes, Diabetes Care 1999; 22: 14081414.

17. Dunger $D B$, Sperling $M A$, Acerini $C L$, Bolin $D J$, Daneman D, Danne TPA, N.S. Glaser, R. Hanas, R.L. Hintz, L.L. Levitsky, M.O. Savage, R.C. Tasker, J.I. Wolsdorf . ESPEMPES consensus statement on diabetic ketoacidosis, Arch Dis Child 2004: 89; 188-194.

18. Kayashima T, Yamaguchi K, Akiyoshi T, Nanimatsu H, Aragaki S, Hosokawa TF. Leukemoid reaction associated with diabetic ketoacidosis - measurement of plasma level of granulocyte colony-stimulating factor. Intern Med 1993; 32: 869-871. 